\title{
Rigid-Earth Nutation Models
}

\author{
J. Souchay \\ Observatoire de Paris, 61 Avenue de l'Observatoire. 75014 Paris, \\ France
}

\begin{abstract}
Despite the fact that the main causes of the differences between the observed Earth nutation and that derived from analytical calculations come from geophysical effects associated with nonrigidity (core flattening, core-mantle interactions, oceans, etc...), efforts have been made recently to compute the nutation of the Earth when it is considered to be a rigid body, giving birth to several "rigid Earth nutation models." The reason for these efforts is that any coefficient of nutation for a realistic Earth (including effects due to nonrigidity) is calculated starting from a coefficient for a rigid-Earth model, using a frequency-dependent transfer function. Therefore it is important to achieve high quality in the determination of rigid-Earth nutation coefficients, in order to isolate the nonrigid effects still not well-modeled.

After reviewing various rigid-Earth nutation models which have been established recently and their relative improvement with respect to older ones, we discuss their specifics and their degree of agreement.
\end{abstract}

\section{Introduction}

The precision in the determination of the Earth's nutation through up-to-date techniques such as Very Long Baseline Interferometry (VLBI) has drastically improved in the recent years, so that it has become usual now to express corrections of the nutation coefficients in terms of microarcseconds, instead of milliarcseconds, as was the case at the eve of the 90s. Comparing the observational data of nutation at this new level of precision, with the theoretical determinations, is of primary interest for the knowledge of our planet. Indeed the way by which the Earth is reacting to the action of the other celestial bodies (the Moon, the Sun and the planets) and thus by which its nutation is modified by external forcing gives precious information on the mechanisms or parameters which cannot be so well known through other studies.

The present conventional nutation series adopted as the IAU 1980 nutation series (Seidelmann, 1982), although being the standard of reference, has been found to differ significantly from observational data obtained by VLBI analyses. For many nutation coefficients, the difference between the estimated value from VLBI analysis and the analytical one is much larger than the uncertainties of the data. Following that fact, an empirical series of nutation for practical use giving close agreement to the data has been elaborated and is now available in the IERS Conventions (McCarthy, 1996). 
Currently active research is devoted to the interpretation of the differences between the observed nutation and its amount as given by the series, and to the construction of series starting from a model as representative as possible, of the various geophysical effects on the nutations. The basic model of nutation of Wahr (1981), applied to an oceanless, elastic, ellipsoidal Earth model derived from the Earth model 1066A of Gilbert and Dziewonski (1975) under the assumption of hydrostatic equilibrium is no longer adequate. An accurate model of nutation must include various effects such as mantle anelasticity, effects of ocean tides and electromagnetic couplings produced at the core-mantle boundary (CMB) or the inner-core boundary (ICB), as was shown by Mathews et al. (1991). Moreover, non-hydrostatic equilibrium has to be considered (Dehant and Defraigne, 1997; Schastok, 1997).

In parallel, the elaboration of these models requires high quality nutation coefficients of the Earth considered as a rigid body, which is a more straightforward and simple approximation. In that frame the challenge is quite clear. It is to model what should be the motion of nutation (and precession) of the Earth considered as a rigid body undergoing the perturbing effects of the Moon, the Sun and the planets, all considered as point mass bodies. Their distances are $a$ priori too large for their forms to have significant effects on the motion. This is a necessary primary step when one wants to compute the coefficients of the nutation of the Earth taking into account various effects related to the oceans, the atmosphere, and all the geophysical influences which have been pointed out in the last paragraph. Thus, until now, all the theories giving birth to the computation of the coefficients of nutation (Wahr, 1981; Dehant and Defraigne, 1997; Mathews et al., 2000; Schastok, 1997; Getino and Ferrándiz, 1999) start from the value of these same coefficients where the Earth is considered as a rigid body. Generally the way to convert from a nutation coefficient for a rigidEarth model, to the same coefficient for a nonrigid Earth, is through a transfer function, generally frequency-dependent that includes various parameters (Love numbers, ellipticity of the core, dynamical ellipticity of the Earth) mainly of geophysical nature.

Thus we understand easily why to determine the coefficients of nutation for a rigid-Earth model is fundamental to reveal in the best way the contributions arising from the nonrigid part associated with the Earth's interior. Moreover, the remarkable improvement of the accuracy of VLBI observations from the beginning of the $80 \mathrm{~s}$ has led to an increasing number of studies devoted to the determination of rigid-Earth nutation. These studies were in particular motivated by the fact that the truncation of the coefficients of nutation of Kinoshita's series for a rigid Earth model (Kinoshita, 1977) that serves as the basic series for the IAU1980 series of nutation (Seidelmann, 1982), was 0.1 mas. This leads to a lack of precision at the level of 1 milliarcsecond for the total nutation amplitude. This shows how it became necessary to push further the level of truncation of the coefficients of rigid-Earth nutation.

Presently the sub-microarcsecond level has been adopted by authors dealing with the topic (Roosbeek and Dehant, 1997; Bretagnon et al., 1999; Souchay et $a l ., 2000)$. A convergence between the rigid-Earth nutation as given by these authors should lead to the conclusion that at least we can be comforted with the validity of such a basic part. In the following we show that this convergence is really satisfactory, after summarizing the evolution of the precision and accuracy 
of the rigid-Earth nutation theory through the various works which have been devoted to the matter in the last decade.

\section{Evolution of the rigid-Earth nutation theory in the 90s}

Kinoshita and Souchay (1990) published new series of nutation for a rigid-Earth model, considering a new level of truncation for the coefficients of nutation: 0.005 mas. This new level made necessary the study of new effects and the extension of some developments. The principal influences can be briefly summed up as (Kinoshita \& Souchay, 1990):

- Influence of the $J_{2}$ harmonic and of the second-order parts of the potential of the Earth $\left(J_{3}\right.$, triaxiality and $\left.J_{4}\right)$,

- Direct and indirect planetary effects,

- Extension of the theory to second order: coupling effect between the rotational motion of the Earth and the orbital motion of the Moon.

\section{New developments in rigid-Earth nutation theory}

The updated value of the general precession in longitude led to a change of the value of the dynamical ellipticity of the Earth as well as the scaling factors for the nutation coefficients relative to the potential of the Moon and the Sun. For this reason, Souchay and Kinoshita $(1996,1997)$ recalculated the coefficients of nutation - due to the lunisolar influence on the geopotential $\left(J_{2}, J_{3}, C_{22}\right.$, $S_{22}$ and $J_{4}$ ) and those coming from the direct torque exerted by the planets - influenced by these changes. Moreover, they presented new contributions to the nutation not included in Kinoshita \& Souchay (1990) such as the influence of the periodic oscillations of the ecliptic, and the planetary tilt-effect. The agreement of the new coefficients of Souchay \& Kinoshita $(1996,1997)$ with those of Hartmann \& Soffel $(1994)$ and Williams $(1994,1995)$ was remarkable.

\section{The effects on nutation of the non-zonal harmonics of third and fourth degree}

Taking into account the new accuracy of the precession-nutation observations, that is to say a few microarseconds, Folgueira et al. $(1998 \mathrm{a}, \mathrm{b})$ calculated the coefficients of the nutation for a rigid-Earth model due to $C_{3 m}, S_{3 m}, C_{4 m}$ and $S_{4 m}(m \neq 0)$ harmonics of the geopotential, which were not considered in previous studies of nutation involving Hamiltonian theory, with a level of truncation of $0.1 \mu a s$ (microarcseconds).

These non-zonal harmonics, together with $C_{22}$ and $S_{22}$, give birth to the short-period nutations, the periods being related to the order $m$ of the corresponding harmonic, that is to say:

- the diurnal terms come from $C_{31}, S_{31}, C_{41}$ and $S_{41}$, 
- the semidiurnal terms are due to the harmonics with $m=2$, and

- the terdiunal terms have their origin in $C_{33}, S_{33}, C_{43}$ and $S_{43}$ harmonics.

The principal diurnal and subdiurnal terms for the nutation in longitude and obliquity are shown in Tables 1 and 2 .

Table 1. Principal terms for quasidiurnal nutations in longitude and obliquity for the figure axis. The unit is $\mu a s$.

\begin{tabular}{|c|c|c|c|c|c|c|c|c|c|c|}
\hline \multicolumn{6}{|c|}{ Argument } & \multirow{2}{*}{ Period } & \multicolumn{2}{|c|}{ Longitude $(\Delta \psi)$} & \multicolumn{2}{|c|}{ Obliquity $(\Delta \varepsilon)$} \\
\hline$\Phi$ & $T_{M}$ & $T_{S}$ & $F$ & $D$ & $\Omega$ & & $\sin$ & $\cos$ & $\sin$ & $\cos$ \\
\hline 1 & 0 & 0 & 1 & 0 & 1 & 0.96215 & -38.2313 & -4.6980 & -1.8567 & 15.1063 \\
\hline 1 & 0 & 0 & -1 & 0 & -1 & 1.03505 & -35.4036 & -4.3513 & -1.5868 & 12.9109 \\
\hline 1 & -1 & 0 & 1 & 0 & 1 & 0.99696 & 24.1443 & 2.9675 & 1.1636 & -9.4678 \\
\hline 1 & 1 & 0 & -1 & 0 & -1 & 0.99758 & -19.9400 & -2.4507 & -0.9716 & 7.9055 \\
\hline 1 & 1 & 0 & 1 & -2 & $\overrightarrow{1}$ & 0.99216 & -7.0629 & -0.8681 & -0.3452 & 2.8089 \\
\hline
\end{tabular}

Table 2. Principal terms for subdiurnal nutations in longitude and obliquity for the figure axis. The unit is $\mu a s$.

\begin{tabular}{|c|c|c|c|c|c|c|c|c|c|c|}
\hline \multicolumn{6}{|c|}{ Argument } & \multirow[t]{2}{*}{ Period } & \multicolumn{2}{|c|}{ Longitude $(\Delta \psi)$} & \multicolumn{2}{|c|}{ Obliquity $(\Delta \varepsilon)$} \\
\hline 15 & $T_{M}$ & $l_{s}$ & $F$ & $D$ & $\Omega$ & & $\sin$ & $\cos$ & $\sin$ & $\cos$ \\
\hline 2 & 0 & 0 & $\mathbf{0}$ & 0 & 0 & 0.49863 & 31.2522 & -17.9397 & -7.1157 & -12.3961 \\
\hline 2 & 0 & 0 & -2 & 0 & -2 & 0.51 & -25.5271 & 14.6533 & 5.6775 & 9.8906 \\
\hline 2 & 0 & 0 & -2 & 2 & -2 & 0.50000 & -10.4455 & 5.9960 & 2.3682 & 4.1255 \\
\hline 2 & -1 & 0 & -2 & 0 & -2 & 0.52743 & -5.0979 & 2.9264 & 1.1818 & 2.0588 \\
\hline 2 & 0 & 0 & -2 & 0 & -1 & 0.51756 & -4.7105 & 2.7040 & 1.0816 & 1.8842 \\
\hline
\end{tabular}

\section{The sub-microarcsecond rigid-Earth nutation series REN 2000}

Souchay et al. (1999) presented the new tables REN 2000 of the nutation of a rigid Earth, starting from Hamiltonian theory, with a sub-microarcsecond level of truncation. All the previously studied influences, together with the secondorder effects due to crossed-nutations and spin-orbit coupling, are included in this paper.

\section{The diurnal and sub-diurnal nutations of REN 2000}

Recently, other rigid-Earth nutation series, calculated using different analytical methods, have come forth. These are SMART97 and RDAN97 series (Bretagnon et al., 1997, 1998; Roosbeek \& Dehant, 1998). Folgueira \& Souchay (1999) and Folgueira et al. (1999) have compared the nutations given by REN 2000 series 
with the corresponding ones in SMART97 and RDAN97. Table 3 gives the comparison for the short-period nutations in the time domain.

Moreover, they have computed corresponding values for a nonrigid Earth of REN 2000 prograde nutations, using a new transfer function developed by Mathews (1999) and showed that some coefficients changed at the level of a few mas. Finally, they have also explained how short-period nutations can perturb the analysis of the polar motion (Bizouard et al., 1999a;b).

Table 3. Comparison in time domain between SMART97, RDAN97 and REN 2000 short period nutations. The unit is $\mu a s$.

\begin{tabular}{lrc}
\hline & $\Delta \psi \sin \varepsilon_{0}$ & $\Delta \varepsilon$ \\
\hline SMART97 - REN 2000 & 1.74 & 3.52 \\
RDAN97 - REN 2000 & 2.40 & 6.80 \\
RDAN97 - SMART97 & 1.40 & 6.80 \\
\hline
\end{tabular}

\section{Conclusions}

In the present paper, we have analyzed the different steps carried out, in this last decade, to reach at the final sub-microarcsecond tables of nutation for a rigid-Earth model REN 2000 (Souchay et al., 1999). We can conclude that:

1. The tables REN 2000 of the nutation for a rigid Earth model, starting from the Hamiltonian theory, catch all the coefficients at a sub-microarcsecond level.

2. There is a good agreement between REN 2000 and other recent works about rigid-Earth nutations (Bretagnon et al., 1997, 1998 ; Roosbeek \& Dehant, 1998).

3. The short-period nutations are the last most important contribution to reach at the 0.1 mas level in the REN 2000 tables.

4. The validity of the analytical series REN 2000 has been checked by a numerical integration of the nutation developed by Souchay \& Kinoshita (1991) and Souchay (1998) and by the study of the residuals between the results given by these two different methods.

Acknowledgments. This investigation was supported by a Postdoctoral Aid for Complutense University of Madrid (Spain) to carry out research work at the Observatory of Paris (France). 


\section{References}

Bizouard, Ch., Folgueira, M. and Souchay, J., 1999a, Proc. Journées 1999. Observatoire de Paris, (submitted).

Bizouard, Ch., Folgueira, M. and Souchay, J., 1999b, Procc. IAU Colloquium 178,613 .

Bretagnon, P., Rocher, P. and Simon, J.-L., 1997, Astron. Astrophys., 318, 305.

Bretagnon, P., Francou, G., Rocher, P. and Simon, J.-L., 1998, Astron. Astrophys., 329, 329.

Dehant V. and Defraigne P., 1997, J. Geophys. Res., 102, 27,659.

Folgueira, M., Souchay, J. and Kinoshita, H., 1998a, Celest. Mech., 69, 373.

Folgueira, M., Souchay, J. and Kinoshita, H., 1998b, Celest. Mech., 70, 147.

Folgueira, M. and Souchay, J., 1999, Proc. Journées 1998. Observatoire de Paris, 178.

Folgueira, M., Bizouard, Ch. and Souchay, J., 1999, Celest. Mech., (submitted). Getino, J. and Ferrándiz, J.M. 1999, Mon. Not. R. Astron. Soc., 306, L45.

Gilbert, F. and A. M. Dziewonski, 1975, Phil. Trans. R. Soc. Lond., A 278, 187.

Hartmann, T. and Soffel, M., 1994, Astron. J., 108, 1115.

Kinoshita, H., 1977, Celest. Mech., 15, 277.

Kinoshita, H. and Souchay, J., 1990, Celest. Mech., 48, 187.

Mathews, P. M., 1999, Proceedings of the Journées Systèmes de Référence Spatio-Temporels 1998, N. Capitaine (ed.), Observatoire de Paris, 161.

Mathews P.M., Herring T.A., Buffett B.A., 2000, J. Geophys. Res., submitted.

Roosbeek, F. and Dehant, V., 1998, Celest. Mech., 70, 215.

Schastok J., 1997, Geophys. J. Int., 130, 137.

Seidelmann, P. K., 1982, Celest. Mech., 27, 79.

Souchay, J. and Kinoshita, H., 1991, Celest. Mech., 52, 45.

Souchay, J. and Kinoshita, H., 1996, Astron. Astrophys., 312, 1017.

Souchay, J. and Kinoshita, H., 1997, Astron. Astrophys., 318, 639.

Souchay, J., 1998, Astron. J., 116, 503.

Souchay, J., Loysel, B., Kinoshita, H. and Folgueira, M., 1999, Astron. Astrophys. Suppl. Ser., 135, 111.

Souchay, J., 1999, Proc. Journées 1998. Observatoire de Paris, 109.

Wahr, J. M., 1981, Geophys. J. R. Astro. Soc., 64, 705.

Williams, J. G., 1994, Astron. J., 108, 711.

Williams, J. G., 1995, Astron. J., 110, 1420. 